\title{
Remarks on Hypo-Elasticity
}

\author{
C. Truesdell
}

(May 22, 1963)

\begin{abstract}
The difference between elasticity and hypo-elasticity is illustrated by calculating explicitly the acoustic tensor for principal waves in an arbitrary hypo-elastic material. It is shown that all principal hypo-elastic waves are necessarily either transverse or longitudinal, just as in an isotropic elastic material, and in distinction to an anisotropic elastic material.
\end{abstract}

When I proposed ${ }^{1}$ the theory of hypo-elasticity, I sought $[1]^{2}$ "a new concept of elastic behavior, mutually exclusive with the theory of finite [elastic] strain except in the linearized case," and I was surprised, although indeed groundlessly, when Noll [2] proved that every isotropic elastic material with invertible stress-strain relation is hypo-elastic. Misled, apparently, by Noll's theorem, Hill and Prager [3] have asserted that every elastic material is hypo-elastic, making hypo-elasticity appear to be a generalization of the classical theory of finite elastic strain. This, certainly, it is not. It embodies a different mathematical concept of the response that in common experience is described as "elastic." Bernstein [4] has set the matter straight by giving a tensorial identity which is a necessary and sufficient condition on the stress field in order that solutions of the equations of hypo-elasticity be consistent with some stress-strain relation. As a most illuminating example he has derived the form of the hypo-elastic constitutive equation satisfied by a perfect fluid and has shown that other solutions of that equation are never compatible with the theory of finite elastic strain. His analysis and example make it clear that different assignments of initial stress for the same hypo-elastic constitutive equation lead to solutions representing behavior so diverse that we can scarcely regard them appropriate to any one physical material. Thus hypo-elasticity seems to offer a convenient summary of certain aspects of response common to many materials, rather than a theory of any particular material.

\footnotetext{
${ }^{1}$ Since wrong statements regarding the origin of hypo-elasticity have been published, its sources will be reviewed here. The basic concept is virtually suggested by Cauchy's theory of initially stressed elastic media (1829), but there is no reference to time rates in his work, which seems to be directed toward infinitesimal static deformation: A.-L. Cauchy, his work, which seems to be directed toward infinitesimal static deformation: A.-L. Cauchy,
Sur l'équilibre et le mouvement intérieur des corps considérés comme des masses continues, Oeuvres 9, 243-269. Special theories of hypo-elastic type, employing invariant tinues, Oeuvres $9,243-269$. Special theories of hypo-elastic type, employing invariant
time rates, were proposed by G. Jaumann in section IX of his Geschlossenes System physikalischer und chemischer Differentialgesetze, Sitzber. Akad. Wiss. Wien (IIa) $120,385-530$ (1911), and in later papers by him and by E. Lohr. The general theory was mentioned by E. Fromm, eq (53a) of his Stoffgesetze des isotropen Kontinuums, insbesondere bei zähplastischen Verhalten, Ing.-Arch. 4, 432-466 (1933), as beig appropriate "zur Darstellung gewisser Erscheinungen der Nachwirkung," but he did not develop its properties. A visco-elastic theory including a special case of hypo-elasticity had been properties. A visco-elastic theory including a special case of hypo-elasticity had been proposed earlier by S. Zaremba, Sur une forme perfectionnee de la theorie de la relaxation, relevance for elastic response.

In the course of exposition of some results obtained by Murnaghan under uncertain and dubious assumptions, I proposed a theory only slightly less general than hypo-elasticity in dubious assumptions, I proposed a theory only slightly less general than hypo-elasticity in
section 56 of the corrections and additions to "The mechanical foundations of elasticity section 56 of the corrections and additions to "The mechanical foundations of elasticity
and fluid dynamics," J. Rational Mech. Anal. 2, 593-616 (1953); at that time I cited the work of Cauchy and Zaremba, and I acknowledged help from Ericksen.

${ }^{2}$ Figures in brackets indicate the literature references at the end of this paper.
}

In this note I wish to correct, by clarifying its origin, the misunderstanding growing from the errors of Hill and Prager and then to illustrate further the difference between hypo-elasticity and elasticity by presenting some new results on hypo-elastic waves.

Elasticity is defined by a stress-strain relation of the form

$$
T^{k m}=\frac{\rho}{\rho_{R}} x^{k},{ }_{\alpha} x^{m},{ }_{\beta} \mathbf{t}^{\alpha \beta}(\mathbf{C}),
$$

where $\mathbf{T}$ is the ordinary Cauchy stress tensor; $\rho$ and $\rho_{R}$ are the densities in the actual configuration and the reference configuration; $x^{k},{ }_{\alpha} \equiv \partial x^{k} / \partial X^{\alpha}, \quad x^{k}=x^{k}$ $(\mathbf{X}, t)$ being the deformation; and $\mathrm{t}$ is a symmetrized function of the components of the symmetric tensor C, where $C_{\alpha \beta}=g_{k m} x^{k},{ }_{\alpha} x^{m},{ }_{\beta}$. In order that the elastic material be isotropic it is necessary and sufficient ${ }^{3}$ that (1) reduce to a relation of the form

$$
\mathbf{T}=\mathbf{f}(\mathbf{B}),
$$

where $\mathbf{f}$ is an isotropic function and where $\mathbf{B}$ has the components $B^{k m}=g^{\alpha \beta} x^{k},{ }_{\alpha} x^{m},{ }_{\beta}$. Differentiating (1) materially with respect to time, we easily show that [5]

$$
\dot{T}^{k m}+T^{k m} D_{q}^{q}-T^{k q} \dot{x}^{m},{ }_{q}-T^{m q} \dot{x}^{k},{ }_{q}=C^{k m p q} D_{p q},
$$

where $\mathbf{x}$ is the velocity field, $\mathbf{D}$ is the stretching tensor $\left(D_{k m} \equiv x_{(k, m)}\right)$, and

$$
\mathrm{C}^{k m p q}=2 \frac{\rho}{\rho_{\mathrm{R}}} x^{k},{ }_{\alpha} x^{m},{ }_{\beta} x^{p}, \gamma x^{q},{ }_{\delta} \frac{\partial \mathrm{t}^{\alpha \beta}}{\partial C_{\gamma \delta}} .
$$

For a given elastic material, the components $C^{k m p q}$ are given functions of the finite strain and rotation.

\footnotetext{
${ }^{3}$ Sufficiency is classical; necessity was proved by F. D. Murnaghan, On the energy of deformation of an elastic solid, Proc. Nat. Acad. Sci. USA 14, 889-891 (1928). Here may lie the source of Prager's error, since he takes (2) as the definition of an elastic material, although in a later passage he obtains for hyperelastic materials a result inconsistent with (2) unless the material is isotropic. The relevant passages occur in sections 1 and 2 of chapter X of Prager's book, cited in footnote 4. It should be noted that Prager's use of the chapter X of Prager's book, cited in footnote 4. It should be noted that Prager's use of the
terms "elastic" and "hyperelastic" differs from that of Noll, who introduced them (op. cit.), terms "elastic" and "hyperelastic" differs from that of Noll, who introduced them (op. cit.),
and of other writers on continuum mechanics. According to Prager's definitions, it can be proved that a hyperelastic material is elastic if and only if it is isotropic. For other writers, hyperelasticity is a special case of elasticity, without exception.

Murnaghan's proof of necessity is unnecessarily elaborate, for the result follows at a glance: since both $\mathbf{T}$ and $\mathbf{B}$ are indifferent, under a rotation $\mathbf{Q}$ they become $\mathbf{Q T Q}^{T}$ and $\mathbf{Q B Q}^{T}$. Hence, if (2) is to hold also in the rotating frame, it is necessary that $\mathbf{Q T}^{T}=\mathbf{Q} \mathbf{f}(\mathbf{B}) \mathbf{Q}^{T}=\mathbf{f}\left(\mathbf{Q B Q} \mathbf{Q}^{7}\right)$.
}

In other words, f must be an isotropic function. 
Hypo-elasticity is defined by an equation of the form ${ }^{4}$

$$
\dot{T}^{k m}-T^{k q} W^{m}{ }_{q}-T^{m q} W_{q}^{k}=\mathrm{H}^{k m p q} D_{p q},
$$

where $\mathbf{W}$ is the spin $\left(W_{k m} \equiv \dot{x}_{[k, m]}\right)$ and where the components $\mathrm{H}^{k m p q}$ are given functions of the stress. Moreover, in order that (5) satisfy the principle of material indifference [6], it is necessary and sufficient that $\mathrm{H}^{k m p q} D_{p q}$ be an isotropic function of $\mathbf{T}$ and $\mathbf{D}$; that is, if we write $\mathbf{H}(\mathbf{T})[\mathbf{D}]$ for the tensor whose components are $\mathrm{H}^{k m p q} D_{p q}$, then

$$
\mathbf{H}\left(\mathbf{Q T Q}^{T}\right)\left[\mathbf{Q D Q}^{T}\right]=\mathbf{Q H}(\mathbf{T})[\mathbf{D}] \mathbf{Q}^{T},
$$

identically in the symmetric tensors $\mathbf{T}$ and $\mathbf{D}$ and the orthogonal tensor $\mathbf{Q}$.

Now both (5) and (3) may be written in the form

$$
\dot{T}^{k m}=\mathrm{D}^{k m p q} \dot{x}_{p, q}
$$

but the functional dependence of $\mathbf{D}$ is different in the two cases. In hypo-elasticity, D is a given function of stress; in elasticity, D depends, in general, also on the strain and rotation from a reference configuration. Elasticity will be included in hypo-elasticity, then, only when the dependence of $\mathbf{D}$ on strain and rotation turns out to be merely apparent. ${ }^{5}$ For an isotropic elastic material, by substituting (2) into (4) it is easy to show that

$$
C^{k m p q}=T^{k m} g^{p q}-T^{k} g^{m p}-T^{m q} g^{k p}+2 \frac{\partial T^{k m}}{\partial B^{p s}} B^{q s} ;
$$

hence $\mathbf{C}$ is a function of $\mathbf{B}$ and $\mathbf{T}$ only. Therefore, if (2) is invertible, $\mathbf{C}$ is equal to a function of $\mathbf{T}$ only, and so is D. Thus Noll's theorem follows. However, the common domain is not exhausted by Noll's theorem, since an easy calculation shows that elastic fluids with invertible pressure functions $p(\rho)$ are always hypo-elastic, although for them the stress-strain relations (2) are never invertible.

However, no material that has a natural state and is anisotropically elastic in small deformation from it is hypo-elastic. As remarked to me by Noll, this fact is most easily seen by putting $\mathbf{T}=0$ in (5). From (6) it follows that $\mathbf{H}(\mathbf{0})[\mathbf{D}]$ is an isotropic linear function

\footnotetext{
${ }^{4}$ We use the flux introduced by Zaremba in 1903, op. cit. footnote 2, and explained by Noll, section 7 of op. cit. footnote 3 and T. Y. Thomas, Kinematically preferred co-ordinate systems, Proc. Nat. Acad. Sci. USA 41, 762-770 (1955). A different motivation for use of this flux was given by G. Jaumann, Elektromagnetische Vorgänge in bewegten Medien, Sitzber. Adad. Wiss. Wien (IIa) 15, 337-390 (1906); see sec. IV. As in my own first work, we could equally well use Cauchy's flux, which stands on the left-hand side of (3). In some recent literature these two fluxes have come to be named, for no good reason, after Jaumann and me, respectively. Any properly formulated rate theory is invariant under change of time flux. It is difficult to discern the objective of the recent literature on the merits and demerits of various time fluxes.

s Here may be the point where the argument of Hill, acited in reference 3, went astray. Hill employed convected coordinates, which confuse the constant metric of space with the changing metric it induces in the body in the course of deformation. The role of the rotation becomes obscure when time rates are calculated in such a system. Apparently Hill concluded that if the relation $t=t(C)$ is invertible, the coefficient-tensor $D$ in (7) becomes a function of $\mathbf{T}$ alone. From the explicit formula (4), valid in any coordinate sys.
tem, we see that this is not so: Even if $\mathbf{C}$ is determined uniquely by $t$ ( $\mathbf{C}$ ), the elasticity $\mathbf{C}$ tem, we see that this is not so: Even if $\mathbf{C}$ is determined uniquely by $\mathbf{t}(\mathbf{C})$, the elasticity $\mathbf{C}$
still depends on both strain and rotation, for anisotropic materials. It is of course impos. sible that the strain and rotation can determine a unique stress $\mathbf{T}$ except when $\mathbf{T}$ is in fact independent of the rotation, for a rotation-dependent stress must certainly vanish for all rotations if it vanishes for one particular one. More generally and precisely, the principle of material indifferences forces the stress to depend on the rotation in the specific, explicit

way implied by (1). (6) must be imposed. However, even with my first and too broad definition, the theorem claimed by Hill and Prager could not be saved, since it reduces to an assertion about the functional dependence $\mathbf{D}$ in (7) for elasticity, irrespective of the invariance properties of $\mathbf{H}$ in hypo-elasticity.
}

of D. Therefore, every hypo-elastic material responds to infinitesimal strain from an unstressed state like an isotropic elastic material.

The domain common to elasticity and hypo-elasticity has thus been nearly delimited. For materials having a natural state, it is no larger than that of isotropic elasticity, at least for small strains; it is no smaller than that of isotropic elasticity with invertible stressrelation; elastic fluids (which of course have neither a natural state nor an invertible stress-strain relation) are included if their pressure functions are invertible.

Bernstein [7] has shown that acceleration waves in a hypo-elastic material, provided the stress be assumed continuous across them, must satisfy a propagation condition of exactly the same form as the elastic one:

$$
\mathbf{Q}(\mathbf{n}) \mathbf{a}=\rho U^{2} \mathbf{a}
$$

where $\mathbf{a}$ is the amplitude and $U$ is the local speed of propagation, and where

$$
Q^{k m}(\mathbf{n})=\mathrm{D}^{k p m q} n_{p} n_{q} .
$$

Let us examine the nature of the acoustical tensor $\mathbf{Q}(\mathbf{n})$. Since $\mathbf{H}(\mathbf{T})[\mathbf{D}]$ is an isotropic function of $\mathbf{T}$ and $\mathbf{D}$ as well as a linear function of $\mathbf{D}$, the fourthorder tensor $\mathbf{H}$ has a representation of the form [8]

$$
\begin{aligned}
& \mathrm{H}^{k m p q}=g^{k m}\left(\boldsymbol{\square}_{1 g^{p q}}+\boldsymbol{\Xi}_{2} T^{p q}+\boldsymbol{\square}_{3} T^{p r} T_{r}^{q}\right) \\
& +T^{k m}\left(\boldsymbol{\Xi}_{4} g^{p q}+\boldsymbol{\Xi}_{5} T^{p q}+\boldsymbol{\Xi}_{6} T^{p r} T_{r}^{q}\right) \\
& +T^{k s} T_{s}^{m}\left(\boldsymbol{\square}_{7} g^{p q}+\boldsymbol{\square}_{8} T^{p q}+\boldsymbol{\square}_{9} T^{p r} T_{r}^{q}\right) \\
& +\boldsymbol{D}_{10 g^{\left(k p_{g} m q\right)}} \\
& +\frac{1}{2} \boldsymbol{\square}_{11}\left(g^{k p} T^{m q}+g^{m p} T^{k q}+g^{k q} \boldsymbol{T}^{m p}+g^{m q} \boldsymbol{T}^{k p}\right) \\
& +\frac{1}{2} \boldsymbol{Q}_{12}\left(g^{k p} T^{m r} T_{r}^{q}+g^{m p} T^{k r} T_{r}^{q}+g^{k q} T^{m r} T_{r}^{p}+g^{m q} T^{k r} T_{r}^{p}\right) \text {. }
\end{aligned}
$$

The coefficients $\boldsymbol{\square}_{\Gamma}$, scalar functions of $\mathbf{T}$, are not uniquely defined, since at most six symmetric secondorder tensors can be linearly independent; however, it is not generally justifiable to set any particular $\boldsymbol{D}_{\Gamma}$ equal to zero. Since, by (5),

$$
\mathrm{D}^{k m p q}=\frac{1}{2}\left(T^{k q_{g} p m}-T^{k m} g^{p q}+T^{p q} g^{k m}-T^{p m} g^{k q}\right)+\mathrm{H}^{k p m q},
$$

(10) is equivalent to

$$
\begin{aligned}
Q^{k m}(\mathbf{n})=\frac{1}{2}\left(g^{k m} T^{p} n_{n_{p}} n_{q}-T^{k m}+T^{k q} n_{q} n^{m}\right. \\
\\
\left.\quad-T^{m q} n_{q} n^{k}\right)+\mathrm{H}^{k p m q} n_{p} n_{q} .
\end{aligned}
$$

Substitution of (11) into (13) yields an explicit if complicated formula for the acoustical tensor for a general direction of propagation, $\mathbf{n}$, in a general hypo-elastic material. 
In the theory of finite strain of isotropic elastic materials, I have defined principal waves as those propagating in the direction of a principal axis of stress [9], and this definition may be adopted also in hypoelasticity. A theorem I proved for isotropic elastic materials may now be extended to all hypo-elastic materials: Every principal wave in a hypo-elastic material is either longitudinal or transverse; indeed, the acoustic axes for principal waves are the principal axes of stress. To prove this, let $\mathbf{n}_{1}$ be a unit vector parallel to the axis corresponding to the principal stress $t_{1}$, so that $T_{m}^{k} n_{1}^{m}=t_{1} n_{1}^{k}$. Then from (13) and (11) we see that

$$
\begin{aligned}
& Q^{k m}\left(\mathbf{n}_{1}\right)=\left[\boldsymbol{\Xi}_{1}+\frac{1}{2} \boldsymbol{\Xi}_{10}+\left(\boldsymbol{\Xi}_{2}+\boldsymbol{\Xi}_{4}+\boldsymbol{\Xi}_{11}\right) t_{1}\right. \\
& +\left(\boldsymbol{\square}_{3}+\boldsymbol{\square}_{5}+\boldsymbol{\square}_{7}+\boldsymbol{\square}_{12}\right) t_{1}^{2} \\
& \left.+\left(\boldsymbol{\Xi}_{6}+\boldsymbol{\Xi}_{8}\right) t_{1}^{3}+\boldsymbol{\square}_{9} t_{1}^{4}\right] n_{1}^{k} n_{1}^{m} \\
& +\frac{1}{2}\left[\boldsymbol{\square}_{10}+\left(\boldsymbol{\Xi}_{11}+1\right) t_{1}+\boldsymbol{\Xi}_{12} t_{1}^{2}\right] g^{k m} \\
& +\frac{1}{2}\left(\boldsymbol{\square}_{11}-1\right) T^{k m}+\frac{1}{2} \boldsymbol{\square}_{12} T^{k r} T_{r}^{m} .
\end{aligned}
$$

The principal axes of this symmetric tensor are the principal axes of stress. The squared speeds of propagation of the corresponding waves may be written down by inspection:

$$
\begin{aligned}
& \rho U_{11}^{2}=Q^{k m}\left(\mathbf{n}_{1}\right) n_{h}^{1} n_{m}^{1}, \\
& =\boldsymbol{\Xi}_{1}+\boldsymbol{\Xi}_{10}+\left(\boldsymbol{\Xi}_{2}+\boldsymbol{\Xi}_{4}+2 \boldsymbol{\Xi}_{11}\right) t_{1} \\
& +\left(\boldsymbol{a}_{3}+\boldsymbol{\square}_{5}+\boldsymbol{\square}_{7}+\boldsymbol{a}_{12}\right) t_{1}^{2} \\
& +\left(\boldsymbol{\square}_{6}+\boldsymbol{\square}_{8}\right) t_{1}^{3}+\boldsymbol{\square}_{9} t_{1}^{4}, \\
& \rho U_{12}^{2}=Q^{k m}\left(\mathbf{n}_{1}\right) n_{k}^{2} n_{m}^{2}, \\
& =\frac{1}{2}\left(t_{1}-t_{2}\right)+\frac{1}{2} \boldsymbol{\square}_{10}+\frac{1}{2} \boldsymbol{\square}_{11}\left(t_{1}+t_{2}\right)+\frac{1}{2} \boldsymbol{\square}_{12}\left(t_{1}^{2}+t_{2}^{2}\right) .
\end{aligned}
$$

These results may be compared with the formulae for the squared speeds of principal waves in isotropic elastic materials [13]. Beyond the identity of the acoustic axes in the two theories, the similarity does not continue. In order to be possible in an isotropic elastic material, the nine squared speeds of propagation, $U_{a l}^{2}$, regarded as functions of the principal stretches, must satisfy numerous conditions of compatibility; if they do satisfy them, they determine the form of the stress-strain relation uniquely. The only conditions of compatibility to be satisfied by the hypo-elastic speeds (15), regarded as functions of the principal stresses, are three identities of the form

$$
\rho\left(U_{12}^{2}-U_{21}^{2}\right)=t_{1}-t_{2}, \ldots,
$$

in addition, of course, to identities expressing invariance under renumbering of axes. The reciprocal theorem (16), stating that the difference of the speeds of transverse waves when amplitude and wave normal are permuted is twice the corresponding principal shear stress divided by the density, is the only property of elastic principal-wave speeds that carries over, in general, to hypo-elastic materials. One cannot even prove that longitudinal waves travel faster than transverse waves in a state of hydrostatic stress. In the theory of elasticity, knowledge of $\mathbf{Q}\left(\mathbf{n}_{a}\right)$ for the three principal directions $\mathbf{n}_{a}$ determines $\mathbf{Q}(\mathbf{n})$ for all $\mathbf{n}$ and hence determines also the form of the stress-strain relations. In hypo-elasticity, there is no indication that the three tensors $\mathbf{Q}\left(\mathbf{n}_{a}\right)$ determine the infinitely many tensors Q(n). Certainly, by (15), knowledge of the nine quantities $U_{a b}^{2}$ as functions of $t_{1}, t_{2}, t_{3}$ does not determine the twelve coefficients $\boldsymbol{D}_{\Gamma}$ as functions of these same variables, but the question is obscured by the indeterminacy of the $\boldsymbol{\Xi}_{\Gamma}$, already remarked.

These results illuminate the nature of hypo-elasticity. It is totally inappropriate for materials regarded physically as "anisotropic." It gives to the physical notion of "elastic isotropy" a mathematical form that is less restrictive than the one embodied in the classical theory of finitely elastic, isotropic materials. In heuristic terms, hypo-elasticity retains the directioal aspects of elastic isotropy while relaxing the relations among magnitudes carried along by the notion of isotropy in the theory of finite strain. It is possible that hypo-elasticity may be appropriate to physical materials that show no sign of preferred states, preferred directions, or fading memory.

\section{References}

[1] C. Truesdell, Hypo-elasticity, J. Rational Mech. Anal. 4, 83133, 1019-1020 (1955), see sec. 3.

[2] W. Noll, On the continuity of the solid and fluid states, J. Rational Mech. Anal. 4, 3-81 (1955), see sec. 156.

[3] R. Hill, Some basic principles in the mechanics of solids without a natural time, J. Mech. Phys. Solids 7, 209-225 (1959), writes on p. 220, "Consequently, an elastic solid is hypo-elastic in Truesdell's sense... This is a simple proof of a theorem of Noll (1955), stated only for isotropic materials." W. Prager in sec. 1 of $\mathrm{ch}$. X of his Introduction to mechanics of continua, Ginn \& Co., 1961 (also Einführung in die Kontinuumsmechanik, Birkhäuser, 1961), writes, "hypo-elastic behavior . . . corresponds to a minimal requirement that a material must fulfill, if it is in any sense to qualify as elastic." The probable sources of these errors are mentioned in footnotes 3 and 5 .

[4] B. Bernstein, Hypo-elasticity and elasticity, Arch. Rational Mech. Anal. 6, 89-104 (1960).

[5] E.q., sec. 55 of my Mechanical Foundations of Elasticity and Fluid Dynamics, J. Rational Mech. Anal. 1, 125-300 (1952).

[6] E.q., sec. 293 of C. Truesdell and R. Toupin's Classical Field Theories, Flügge's Encyclopedia of Physics 3, 226-793 (1960).

[7] B. Bernstein, Conditions for second-order waves in hypoelasticity, Trans. Soc. Rheol. 6, 263-273 (1962). Similar results, under assumptions partly more general and partly more special, were obtained by Hill, Acceleration waves in solids, J. Mech. Phys. Solids 10, 1-16 (1962).

[8] This representation follows by specializing more general results derived by R. S. Rivlin and J. L. Ericksen in sections 40 and 33 of Stress-deformation relations for isotropic materials, J. Rational Mech. Anal. 4, 323-425 (1955).

[9] C. Truesdell, General and exact theory of waves in finite elastic strain, Arch. Rational Mech. Anal. 8, 263-296 (1961), see sec. 10 .

(Paper 67B3-98) 